\title{
Parametrização e avaliação do modelo DSSAT/Canegro para variedades brasileiras de cana-de-açúcar
}

\author{
Daniel Silveira Pinto Nassif(1), Fabio Ricardo Marin(2), Wander José Pallone Filho(3), Ronaldo Souza Resende(4) \\ e Giampaolo Queiroz Pellegrino(2)
}

(1)Universidade de São Paulo, Escola Superior de Agricultura Luiz de Queiroz, Avenida Pádua Dias, no 11, CEP 13418-900 Piracicaba, SP. E-mail: dspnassif@usp.br (2)Embrapa Informática Agropecuária, Avenida André Tosello, no 209, CEP 13083-886 Campinas, SP. E-mail: marin@cnptia.embrapa.br, giam@cnptia.embrapa.br ${ }^{(3)}$ Centro de Tecnologia Canavieira, Fazenda Santo Antonio, s/no, CEP 13400-970 Piracicaba, SP. E-mail: wander@ctc.com.br ${ }^{(4)}$ Embrapa Tabuleiros Costeiros, Avenida Beira Mar, no 3.250, CEP 49025-040 Aracaju, SE. E-mail: ronaldo@cpatc.embrapa.br

\begin{abstract}
Resumo - O objetivo deste trabalho foi parametrizar e avaliar o modelo DSSAT/Canegro para cinco variedades brasileiras de cana-de-açúcar. A parametrização foi realizada a partir do uso de dados biométricos e de crescimento das variedades CTC 4, CTC 7, CTC 20, RB 86-7515 e RB 83-5486, obtidos em cinco localidades brasileiras. Foi realizada análise de sensibilidade local para os principais parâmetros. A parametrização do modelo foi feita por meio da técnica de estimativa da incerteza de probabilidade generalizada ("generalized likelihood uncertainty estimation", Glue). Para a avaliação das predições, foram utilizados, como indicadores estatísticos, o coeficiente de determinação $\left(\mathrm{R}^{2}\right)$, o índice $\mathrm{D}$ de Willmott e a raiz quadrada do erro-médio (RMSE). As variedades CTC apresentaram índice D entre 0,870 e 0,944, para índice de área foliar, altura de colmo, perfilhamento e teor de sacarose. A variedade RB 83-5486 apresentou resultados similares para teor de sacarose e massa de matéria fresca do colmo, enquanto a variedade RB 86-7515 apresentou valores entre 0,665 e 0,873 , para as variáveis avaliadas.
\end{abstract}

Termos para indexação: Saccharum, biometria, calibração, modelagem, validação.

\section{Parameterization and evaluation of the DSSAT/Canegro model for Brazilian sugarcane varieties}

\begin{abstract}
The objective of this work was to parameterize and to evaluate the DSSAT/Canegro model for five Brazilian sugarcane varieties. The parameterization was done using biometric and growth data from the varieties CTC 4, CTC 7, CTC 20, RB 86-7515, and RB 83-5486, obtained in five Brazilian locations. Local sensitivity analysis was performed for the main parameters. Model parameterization was done using the generalized likelihood uncertainty estimation (Glue) method. The predictions were evaluated using the coefficient of determination $\left(\mathrm{R}^{2}\right)$, Willmott's D-index, and the root mean square error (RMSE) as statistical indexes. CTC varieties showed D values ranging from 0.870 to 0.944 for leaf area index, stalk height, tillering, and sucrose content. The RB 83-5486 variety showed similar results for sucrose content and fresh matter mass of stalks, whereas the RB 86-7515 variety showed values ranging from 0.665 to 0.873 for all variables analyzed.
\end{abstract}

Index terms: Saccharum, biometrics, calibration, modelling, validation.

\section{Introdução}

Acultura da cana-de-açúcar(Saccharum officinarum L.) tem importância econômica, social e ambiental para o Brasil. O etanol brasileiro de cana-de-açúcar atrai investimentos internos e externos, e é considerado exemplo de programa de energia renovável entre os países emergentes (Goldemberg, 2007). A ampliação desses investimentos exige planejamento estratégico da expansão da cultura da cana-de-açúcar no país, e modelos de simulação baseados em processos fisiológicos podem ajudar nessa tarefa.
Modelos de crescimento e desenvolvimento de culturas baseados em processos podem contribuir no monitoramento e na previsão de produtividade, assim como auxiliar na compreensão dos mecanismos envolvidos nas respostas da cultura ao ambiente (Marin et al., 2011). Existem diversos modelos de simulação de crescimento da cana-de-açúcar em uso atualmente, como: Auscane (Jones et al., 1988), QCane (Liu \& Kingston, 1995), APSIM(Keating et al., 1999), Mosicas (Martiné, 2003) e Casupro (Villegas et al., 2005). Entre esses modelos, o DSSAT/Canegro (Inman-Bamber, 1991; Singels et al., 2008) é um dos principais. 
O modelo DSSAT/Canegro, baseado no modelo Ceres-Maize (Jones \& Kiniry, 1986), foi desenvolvido na África do Sul para modelar os processos fisiológicos mais relevantes da indústria açucareira sul-africana (Inman-Bamber, 1991). Incluído no conjunto de modelos "decision support system for agrotechnology transfer" (DSSAT versão 3.1) (Inman-Bamber \& Kiker, 1997) e atualizado na versão 4.5 do DSSAT (Singels et al., 2008), o DSSAT/Canegro vem sendo aplicado em diversas regiões do mundo para análise e avanço no conhecimento dos sistemas de produção de cana-de-açúcar (Inman-Bamber, 1994; Singels \& Bezuidenhout, 2002; Knox et al., 2010; Singels et al., 2010; Marin et al., 2011).

A utilização de modelos de crescimento de plantas em condições diferentes do local onde eles foram desenvolvidos requer testes e adaptações, em virtude das diferenças de solo, clima e características genéticas das culturas. O modelo DSSAT/Canegro, por ter sido desenvolvido para variedades sul-africanas, foi inicialmente calibrado com duas variedades para os sistemas produtivos do Centro-Sul do Brasil (Marin et al., 2011). A despeito deste trabalho inicial, é imprescindível que se avalie o modelo com mais variedades, sistemas produtivos e zonas agroecológicas do país, e que se analise seu desempenho, seus aspectos favoráveis, suas limitações e seus pontos passíveis de melhoria.

O objetivo deste trabalho foi parametrizar e avaliar o modelo DSSAT/Canegro para cinco variedades brasileiras de cana-de-açúcar.

\section{Material e Métodos}

A parametrização e a validação do modelo DSSAT/ Canegro foram feitas a partir de dados experimentais, para as variedades CTC 4, CTC 7 e CTC 20, e com a utilização dos dados biométricos descritos por Pellegrino (2001), Costa et al. (2007) e Silva (2007), para as variedades RB 86-7515 e RB 83-5486. Os experimentos foram conduzidos em Pradópolis, SP $\left(21^{\circ} 18^{\prime} 24^{\prime \prime} S\right.$ e $48^{\circ} 05^{\prime} 31^{\prime \prime} \mathrm{W}$, a $608 \mathrm{~m}$ de altitude) e Piracicaba, SP $\left(22^{\circ} 42^{\prime} 30^{\prime \prime} \mathrm{S}\right.$ e $47^{\circ} 38^{\prime} 00^{\prime \prime} \mathrm{W}$, a $546 \mathrm{~m}$ de altitude). Ambos os locais apresentam clima Cwa, conforme a classificação de Köppen. $\mathrm{O}$ solo em Pradópolis foi classificado como Latossolo Vermelho eutroférrico, de textura argilosa, e em Piracicaba, como Latossolo Vermelho, de textura argilosa. Foram obtidos dados meteorológicos de temperaturas máxima e mínima $\left({ }^{\circ} \mathrm{C}\right)$, radiação global $\left(\mathrm{W} \mathrm{m}^{-2} \mathrm{D}^{-1}\right)$ e precipitação $(\mathrm{mm})$, por meio de estações automáticas localizadas a, aproximadamente, 1,0 km de distância dos locais dos experimentos, nas duas localidades.

O modelo DSSAT/Canegro utiliza dados meteorológicos diários (radiação solar, temperaturas máxima e mínima, e precipitação) e dados de balanço hídrico como entrada, com base em processos de modelagem do crescimento e do desenvolvimento da cana-de-açúcar, incluindo fenologia, desenvolvimento do dossel, acúmulo de biomassa e sacarose, particionamento, crescimento de raízes, estresse hídrico e acamamento (Singels et al., 2008). O modelo também requer dados de entrada de solo (capacidade de campo, ponto de murcha permanente, saturação de água e profundidade do solo) que regulam o balanço hídrico (Ritchie, 1998).

Utilizou-se o delineamento experimental de blocos ao acaso, com quatro repetições de cada cultivar, formadas por seis linhas de plantio de $15 \mathrm{~m}$ de comprimento, nas quais os dados foram obtidos na terceira, quarta $\mathrm{e}$ quinta linhas, nos 5,0 m centrais. Foram marcadas dez plantas por parcela, para acompanhamento das medidas biométricas. O plantio em Pradópolis foi realizado em $5 / 3 / 2009$, com espaçamento entre linhas de $1,5 \mathrm{~m} \mathrm{e}$ adubação e tratos culturais adequados. A colheita foi realizada em 2/7/2010. Em Piracicaba, o plantio foi realizado em 15/4/2009, e a colheita foi realizada em 24/8/2010.

Foram coletados dados do número de perfilhos por metro quadrado, altura de colmo (metros), número de folhas verdes por planta e índice de área foliar (IAF), além de análise tecnológica do teor de sacarose e das massas de matéria fresca e seca do colmo.

Procedeu-se à análise de sensibilidade local para determinar quais os parâmetros a serem otimizados. Os parâmetros foram avaliados pelos resultados de quatro variáveis de saída: IAF máximo de folhas verdes, massa de matéria fresca de colmo $\left(\mathrm{Mg} \mathrm{ha}^{-1}\right)$, massa de matéria seca da parte aérea $\left(\mathrm{Mg} \mathrm{ha}^{-1}\right)$ e altura do colmo (metros). Os parâmetros de entrada (Tabela 1), quando conveniente, tiveram variações individuais de -10 e $+10 \%$, e os efeitos foram analisados pelo cálculo do índice de sensibilidade relativa (Saltelli, 2002).

Para parametrização, utilizou-se um método objetivo com uso de programa otimizador de parâmetros, baseado na técnica de estimativa da incerteza de probabilidade 
generalizada ("generalized likelihood uncertainty estimation", Glue) (Mertens et al., 2004). De acordo com essa técnica, a melhor combinação de parâmetros é selecionada pela comparação das estimativas do modelo com os dados observados em condições de campo. O algoritmo contendo a Glue utilizada no presente trabalho valia-se apenas dos dados finais do ciclo da cultura e desprezava os dados coletados durante o seu desenvolvimento. $\mathrm{O}$ procedimento de ajuste da Glue foi feito em aproximações sucessivas, tendo-se restringido a faixa de ajuste dos parâmetros a cada aproximação. Cada rodada de parametrização por meio da ferramenta Glue foi feita com uso de cinco mil iterações por cada variedade analisada (Jones et al., 2010). O melhor ajuste do modelo, para cada variedade, foi definido com base no coeficiente de determinação $\left(\mathrm{R}^{2}\right)$, no índice D de Willmott (1982) e na raiz quadrada do erro-médio (RMSE).

A avaliação do modelo foi realizada com dados dos campos experimentais de Piracicaba, para as variedades CTC 4, CTC 7 e CTC 20, dados bibliográficos de Costa et al. (2007), para a variedade RB 83-5486, e dados de Silva (2007), para a variedade RB 86-7515. Para verificação da qualidade da parametrização, foram utilizados a análise de regressão linear, os índices $\mathrm{R}^{2} \mathrm{e}$
D de Wilmott (1982), e a RMSE. A variedade padrão NCo 376 foi utilizada para comparação das midificações nos parâmetros, após a calibração do modelo DSSAT/ Caneagro.

\section{Resultados e Discussão}

De modo geral, as cultivares CTC 4, CTC 7 e CTC 20 apresentaram padrão de desenvolvimento similar ao longo do período de coleta de dados, no experimento em Pradópolis. A variedade CTC 7 apresentou menor IAF máximo $(2,7)$ e menor número de perfilhos durante o ciclo, com pico de perfilhamento de 16,6 perfilhos $\mathrm{m}^{-2}$ e estabilização em 8,4 perfilhos $\mathrm{m}^{-2}$. Contudo, essa variedade apresentou maior altura de colmo desde o início do crescimento até o final do ciclo. As variedades CTC 4 e CTC 20 apresentaram IAF, perfilhamento e altura de colmos semelhantes, que chegaram a valores máximos em torno de 3,3. A CTC 4 apresentou menor número de folhas verdes, com média de 7,2, enquanto a CTC 7 e a CTC 20 apresentaram em torno de nove folhas verdes por planta ao longo do ciclo.

A produtividade média do experimento em Pradópolis foi de 140,3 $\mathrm{Mg} \mathrm{ha}^{-1}$, sendo a CTC 20 a

Tabela 1. Descrição de parâmetros de cultivares e de unidades do modelo DSSAT/Canegro.

\begin{tabular}{|c|c|c|}
\hline Parâmetro & Unidade & Descrição \\
\hline Parce $_{\text {máx }}$ & $\mathrm{g} \mathrm{MJ}^{-1}$ & $\begin{array}{c}\text { Máxima eficiência na conversão de radiação expressa em assimilados produzidos antes da respiração, por unidade } \\
\text { de radiação fotossinteticamente ativa (PAR) }\end{array}$ \\
\hline APFMX & $\mathrm{Mg} \mathrm{Mg}^{-1}$ & Fração máxima de incremento de massa de matéria seca que pode ser alocado para massa de matéria seca da parte aérea \\
\hline $\mathrm{STKPF}_{\text {máx }}$ & $\mathrm{Mg} \mathrm{Mg}^{-1}$ & $\begin{array}{c}\text { Fração do incremento diário de massa de matéria seca aérea particionada para o colmo em altas temperaturas } \\
\text { em cultura madura }\end{array}$ \\
\hline Suca & $\mathrm{Mg} \mathrm{Mg}^{-1}$ & Máxima sacarose contida na base do colmo \\
\hline TBFT & ${ }^{\circ} \mathrm{C}$ & Temperatura em que o particionamento para sacarose é $50 \%$ do máximo incremento de massa de colmo sem estresse \\
\hline Tthalfo & GD & Graus-dia para que o desenvolvimento do dossel atinja metade do espaçamento \\
\hline Tbase & ${ }^{\circ} \mathrm{C}$ & Temperatura base para o desenvolvimento do dossel \\
\hline $\mathrm{LF}_{\text {máx }}$ & Folhas & $\begin{array}{l}\text { Número máximo de folhas verdes saudáveis (plantas adequadamente irrigadas serão velhas o suficiente } \\
\text { para perderem folhas após este número) }\end{array}$ \\
\hline MXLFArea & $\mathrm{cm}^{2}$ & Área foliar máxima atribuída às folhas sobre o número de folhas MXLFArno \\
\hline MXLFArno & Folha & Número da folha acima do qual a área foliar é limitada pelo MXLFArea \\
\hline P11 & GD & Intervalo de filocrono 1 para as folhas abaixo do Pswitch \\
\hline $\mathrm{P} 12$ & GD & Intervalo de filocrono 2 para as folhas acima do Pswitch \\
\hline Pswitch & Folha & Número da folha em que há mudança no filocrono \\
\hline TTPLNTEM & GD & Graus-dia para emergência da cana-planta \\
\hline TTRATNEM & GD & Graus-dia para emergência da soqueira \\
\hline ChupiBase & GD & Graus-dia para início do crescimento do colmo \\
\hline TT_PopGrowth & GD & Graus-dia para ocorrer o pico do perfilhamento \\
\hline Max_Pop & Colmos $\mathrm{m}^{-2}$ & População de perfilhos máxima \\
\hline PopTT16 & Colmos $\mathrm{m}^{-2}$ & População de colmos após 1.600 graus-dia \\
\hline LG_AMBase & $\mathrm{Mg} \mathrm{ha}^{-1}$ & Massa de matéria fresca da parte aérea onde ocorre início do acamamento \\
\hline
\end{tabular}


mais produtiva, com $146 \mathrm{Mg} \mathrm{ha}^{-1}$, e com maior teor de sacarose $(16 \%)$. As variedades CTC 4 e CTC 7 apresentaram teor de sacarose próximos, em torno de $15,5 \%$. A produtividade mais elevada da variedade CTC 20 pode estar relacionada ao IAF mais elevado e também ao seu maior número de perfilhos. Nesse sentido, a variedade CTC 4 apresentou menor produtividade, aparentemente, em razão do menor IAF e da menor capacidade de perfilhamento. Cabe destacar que a produtividade de sacarose da variedade CTC 20 foi de aproximadamente $23,5 \mathrm{Mg} \mathrm{ha}^{-1}$.

No experimento em Piracicaba, também foram observados padrões de desenvolvimento similares entre as variedades CTC, mas a CTC 4 apresentou menor IAF máximo $(2,41)$ e menor perfilhamento. A variedade CTC 20 apresentou maior IAF $(3,68)$ e maior pico de perfilhamento (19,1 perfilhos $\left.\mathrm{m}^{-2}\right)$, o que pode explicar a maior produtividade obtida com essa variedade (148 $\left.\mathrm{Mg} \mathrm{ha}^{-1}\right)$. A CTC 7 apresentou comportamento semelhante ao da CTC 20, porém com perfilhamento menos intenso ( 9 perfilhos $\mathrm{m}^{-2}$ ) na maturidade, o que provavelmente está relacionado à menor produtividade $\left(136 \mathrm{Mg} \mathrm{ha}^{-1}\right)$. O número de folhas verdes por planta praticamente não diferiu entre as três variedades, tendo ficado em torno de 5,5 folhas verdes por planta, em média, com pico e estabilização em sete folhas verdes por planta.

$\mathrm{O}$ teor de sacarose das três variedades foi praticamente o mesmo na colheita, em torno de $17 \%$. Portanto, nota-se que as diferenças das variáveis biométricas pouco influenciaram o acúmulo de sacarose das variedades. No entanto, ao se extrapolar esse resultado para a produção de sacarose por unidade de área, verificou-se que a variedade CTC 20 também foi a mais produtiva, com aproximadamente $25 \mathrm{Mg}$ ha $^{-1}$ de sacarose.

$\mathrm{Na}$ análise de sensibilidade local, os parâmetros

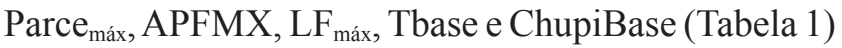
apresentaram os maiores valores de sensibilidade relativa (SR), de 1,04 a 1,57, sendo que o parâmetro APFMX apresentou relativa importância nas quatro variáveis de saída avaliadas.

Os valores utilizados na parametrização (Tabela 2), gerados por meio da ferramenta de otimização Glue, diferiram em grande parte dos valores padrão do DSSAT/Canegro com a variedade NCo 376. Observou-se que o parâmetro MXLFArea aumentou em aproximadamente $30 \%$, em comparação ao padrão, o que corrobora dados obtidos por Marin et al. (2011), que também encontraram valores para esse parâmetro em torno de $50 \%$ maiores que o padrão. Além disso, verificou-se decréscimo nos parâmetros Max_Pop e PopTT16, que controlam a população de perfilhos, semelhantemente ao ocorrido com as cinco variedades avaliadas, em que o máximo número de perfilhos ficou em torno de 19 perfilhos $\mathrm{m}^{-2}$ - quase $40 \%$ menor do que o padrão -, e o número de perfilhos na maturidade em torno de 9,0 perfilhos $\mathrm{m}^{-2}$ - cerca de $30 \%$ menor.

No parâmetro de acúmulo de sacarose (Suca), houve aumento em todas as variedades brasileiras, quando comparadas à NCo 376 padrão, ao se realizar a simulação com valores padrão. $\mathrm{O}$ teor de sacarose ficou subestimado para as cinco variedades brasileiras.

As variedades CTC 7, CTC 20 e RB 83-5486 apresentaram os melhores resultados de parametrização para a variável IAF de folhas verdes, tendo sido obtidos $\mathrm{R}^{2}$ de $0,71,0,63$ e 0,76 e valores de índice $\mathrm{D}$ de 0,75 , 0,85 e 0,85 , respectivamente. Os dados da variedade CTC 4, apresentaram baixo coeficiente de determinação $\left(\mathrm{R}^{2}=0,21\right)$, mas bom índice de concordância $(\mathrm{D}=0,68)$. A RMSE, para as variedades CTC, ficou abaixo de $1,0 \mathrm{~m}^{2} \mathrm{~m}^{-2}$, enquanto para a RB 83-5486 foi de $1,43 \mathrm{~m}^{2} \mathrm{~m}^{-2}$.

Tabela 2. Valores dos parâmetros de cultivares utilizados na parametrização do modelo DSSAT/Canegro, para as variedades avaliadas CTC 4, CTC 7, CTC 20, RB 86-7515 e RB 83-5486, e para a variedade padrão NCo 376.

\begin{tabular}{lcccccc}
\hline Parâmetro & CTC & CTC & CTC & RB & RB & NCo \\
& 4 & 7 & 20 & $86-7515$ & $83-5486$ & 376 \\
\hline Parce $_{\text {máx }}$ & 11,99 & 11,78 & 12,50 & 12,86 & 13,52 & 9,90 \\
APFMX & 0,884 & 0,883 & 0,889 & 0,843 & 0,865 & 0,88 \\
STKPF $_{\text {máx }}$ & 0,700 & 0,699 & 0,700 & 0,699 & 0,760 & 0,650 \\
Suca & 0,689 & 0,690 & 0,695 & 0,680 & 0,695 & 0,580 \\
TBFT & 25 & 26 & 26 & 25 & 26 & 25 \\
Tthalfo & 230,4 & 229,6 & 225,6 & 250,8 & 275,8 & 250,0 \\
Tbase & 14,49 & 15,00 & 15,62 & 15,71 & 15,62 & 16,00 \\
LF $_{\text {máx }}$ & 9,924 & 9,969 & 10,880 & 9,960 & 9,518 & 12,000 \\
MXLFArea & 500,2 & 515,6 & 520,9 & 500,2 & 500,9 & 360,0 \\
MXLFArno & 14,99 & 14,80 & 15,00 & 17,19 & 15,35 & 15,00 \\
P11 & 89,3 & 91,2 & 90,9 & 89,0 & 90,1 & 69,0 \\
P12 & 120,1 & 125,5 & 120,9 & 150,0 & 149,4 & 169,0 \\
Pswitch & 15,90 & 15,02 & 15,02 & 16,14 & 16,33 & 18,00 \\
TTPLNTEM & 500,6 & 200,9 & 200,9 & 300,4 & 509,4 & 428,0 \\
TTRATNEM & 200,5 & 205,9 & 265,3 & 290,9 & 211,4 & 203,0 \\
ChupiBase & 850,2 & 890,6 & 850,2 & 855,0 & 547,6 & $1.050,0$ \\
TT_PopGrowth & 559,8 & 620,7 & 610,1 & 650,4 & 530,2 & 600,0 \\
Max_Pop & 18,57 & 16,75 & 19,50 & 20,35 & 19,62 & 30,00 \\
PopTT16 & 9,446 & 8,995 & 9,952 & 8,190 & 9,556 & 13,300 \\
LG_AMBase & 220 & 220 & 220 & 220 & 220 & 220 \\
\hline
\end{tabular}


Quanto à variável de perfilhamento, as três variedades CTC apresentaram boa parametrização. A variedade CTC 20 apresentou valores de $\mathrm{R}^{2}$ e D maiores $(0,88$ e 0,94 , respectivamente), e a CTC 7 índices mais baixos $(0,63$ e 0,77$)$.

A variável número de folhas verdes por planta apresentou os índices estatísticos mais baixos, para as três variedades CTC avaliadas. Esse resultado é similar ao observado por Marin et al. (2011) e parece estar mais relacionado à relativa estabilidade temporal da variável ao longo do ciclo do que à deficiência do modelo em predizê-la. Na parametrização, obtiveramse valores de $\mathrm{R}^{2}$ de 0,01 (para CTC 4 e CTC 7) a 0,1 (para CTC 20), enquanto o índice D variou de 0,21 a 0,49 .

Com índices estatísticos de $\mathrm{R}^{2}$ entre 0,89 e 0,97 , e de D entre 0,75 e 0,88 , a variável altura de colmo apresentou a melhor parametrização para as variedades CTC e RB 86-7515, indício da boa habilidade do modelo DSSAT/Canegro em predizer esta variável.

Para as variedades CTC 7 e CTC 20, houve boa correlação entre os dados observados e estimados após a parametrização, com $R^{2}=0,75$ e 0,81 , respectivamente. Porém, o índice D, que ficou entre 0,43 e 0,5 , para as três variedades, indicou baixa acurácia. Na CTC 4, além de baixa acurácia, os valores apresentaram baixo coeficiente de determinação $\left(\mathrm{R}^{2}=0,36\right)$.

Para as variáveis de massa de matéria fresca e seca do colmo, somente a variedade RB 83-5486 foi avaliada na parametrização, por apresentar dados observados durante todo o ciclo. Os índices estatísticos indicaram boa correlação e concordância em ambas as variáveis, em torno de $\mathrm{R}^{2}=0,5$ e $\mathrm{D}=0,7$.

$\mathrm{Na}$ avaliação do modelo parametrizado, a variedade RB 86-7515 apresentou superestimativa no acúmulo de matéria fresca durante todo o ciclo; porém, o valor final de produção de matéria fresca apresentou-se compatível com os dados observados, o que ocorreu com as três variedades CTC e também com a RB 83-5486. Para a RB 86-7515, obteve-se $\mathrm{R}^{2}=0,507$ e $\mathrm{D}=0,743$, com $\mathrm{RMSE}=30,3 \mathrm{Mg} \mathrm{ha}^{-1}$ (Tabela 3). Esses valores diferiram dos encontrados por O'Leary (2000), para a variedade NCo 376: $\mathrm{R}^{2}=0,73$ e RMSE $=11,11 \mathrm{Mg} \mathrm{ha}^{-1}$. As variedades CTC 4, CTC 7, CTC 20 e RB 83-5486 apresentaram produtividade final próxima à observada em campo.

Para as quatro variedades avaliadas, a estimativa do IAF de folhas verdes apresentou atraso em seu início, em comparação aos dados obtidos, e atingiu pico em torno de 3,5, aos 250 dias após plantio (DAP), tendo-se mantido estável durante o ciclo da cultura e decaído ao final. Suguitani (2006) obteve RMSE $=0,59\left(\mathrm{~m}^{2} \mathrm{~m}^{-2}\right) \mathrm{e}$ valores de $\mathrm{R}^{2}$ maiores que 0,92 , para quatro variedades, com as estimativas realizadas com o modelo Mosicas (Martiné, 2003).

As variedades CTC 4, CTC 7 e CTC 20 apresentaram boa correlação com os dados validados (Tabela 3 ) e geraram valores de $\mathrm{R}^{2}$ próximos a 1,0 e elevados também para D. Esses resultados foram melhores que os obtidos por Inman-Bamber (1991), que utilizou uma versão preliminar do Canegro: $\mathrm{R}^{2}=0,43$ e $\mathrm{D}=0,80$. Contudo, os valores observados no presente trabalho

Tabela 3. Valor dos parâmetros estatísticos avaliados na validação do modelo DSSTA/Canegro, para as variáveis altura, número de perfilhos, índice de área foliar, teor de sacarose, número de folhas verdes, e massa de matéria seca e fresca de colmo, para as variedades avaliadas.

\begin{tabular}{|c|c|c|c|c|c|}
\hline Parâmetro $^{(1)}$ & $\begin{array}{c}\text { CTC } \\
4\end{array}$ & $\begin{array}{c}\text { CTC } \\
7 \\
\end{array}$ & $\begin{array}{c}\text { CTC } \\
20 \\
\end{array}$ & $\begin{array}{c}\mathrm{RB} \\
86-7515 \\
\end{array}$ & $\begin{array}{c}\mathrm{RB} \\
83-5486 \\
\end{array}$ \\
\hline & \multicolumn{5}{|c|}{ Altura } \\
\hline $\mathrm{R}^{2}$ & 0,96 & 0,96 & 0,98 & 0,70 & $-(2)$ \\
\hline $\mathrm{D}$ & 0,77 & 0,85 & 0,72 & 0,82 & - \\
\hline \multirow[t]{2}{*}{ RMSE } & 0,43 & 0,43 & 0,59 & 0,59 & - \\
\hline & \multicolumn{5}{|c|}{ Perfilhos } \\
\hline $\mathrm{R}^{2}$ & 0,89 & 0,72 & 0,92 & 0,58 & - \\
\hline $\mathrm{D}$ & 0,80 & 0,83 & 0,95 & 0,87 & - \\
\hline \multirow[t]{2}{*}{ RMSE } & 4,88 & 3,82 & 2,72 & 3,31 & - \\
\hline & \multicolumn{5}{|c|}{ Índice de área foliar } \\
\hline $\mathrm{R}^{2}$ & 0,98 & 0,92 & 0,99 & - & - \\
\hline $\mathrm{D}$ & 0,94 & 0,87 & 0,93 & - & - \\
\hline \multirow[t]{2}{*}{ RMSE } & 0,42 & 0,73 & 0,64 & - & - \\
\hline & \multicolumn{5}{|c|}{ Sacarose } \\
\hline $\mathrm{R}^{2}$ & 0,81 & 0,75 & 0,51 & - & - \\
\hline $\mathrm{D}$ & 0,55 & 0,47 & 0,49 & - & - \\
\hline \multirow[t]{2}{*}{ RMSE } & 2,06 & 1,44 & 2,61 & - & - \\
\hline & \multicolumn{5}{|c|}{ Folhas verdes por planta } \\
\hline $\mathrm{R}^{2}$ & 0,94 & 0,48 & 0,74 & - & - \\
\hline $\mathrm{D}$ & 0,61 & 0,59 & 0,80 & - & - \\
\hline \multirow[t]{2}{*}{ RMSE } & 3,16 & 2,33 & 1,67 & - & - \\
\hline & \multicolumn{5}{|c|}{ Massa de matéria seca } \\
\hline $\mathrm{R}^{2}$ & - & - & - & 0,53 & - \\
\hline $\mathrm{D}$ & - & - & - & 0,67 & - \\
\hline \multirow[t]{2}{*}{ RMSE } & - & - & - & 9,51 & - \\
\hline & \multicolumn{5}{|c|}{ Massa de matéria fresca } \\
\hline $\mathrm{R}^{2}$ & - & - & - & - & 0,51 \\
\hline $\mathrm{D}$ & - & - & - & - & 0,74 \\
\hline RMSE & - & - & - & - & 30,36 \\
\hline
\end{tabular}

${ }^{(1)} \mathrm{R}^{2}$, coeficiente de determinação; D, índice D de Willmott; RMSE, raiz quadrada do erro-médio. ${ }^{(2)}$ Dados não disponíveis. 
ficaram próximos aos obtidos por Suguitani (2006) com o modelo Mosicas, com $\mathrm{R}^{2}>0,92$.

Para a variável altura de colmo, houve boa correlação entre os dados observados e os estimados, com início do crescimento do colmo das três variedades CTC aproximadamente aos 50 DAP, e da variedade RB 86-7515 em torno de 25 DAP. Nesta última, houve superestimativa inicial do crescimento do colmo, o que se inverteu, aos 300 DAP, quando o modelo passou a subestimar os dados observados.

Houve correlação entre os dados observados e estimados, com $\mathrm{R}^{2}$ entre 0,701 e 0,821 , e $\mathrm{D}$ entre 0,720 e 0,853 (Tabela 3). Esses resultados são comparáveis aos obtidos por Suguitani (2006), com o modelo Mosicas. Entretanto, este autor verificou RMSE acima de $7,47 \mathrm{~m}$, tendo chegado a $17,1 \mathrm{~m}$, o que representa mais de dez vezes a RMSE obtida para as variedades CTC analisadas no presente trabalho.

A parametrização utilizada para a variedade CTC 4 acarretou atraso na simulação do início do perfilhamento, com pico aos 120 DAP e estabilização aos 250 DAP, em cerca de 9,0 perfilhos $\mathrm{m}^{-2}$, o que coincide com os dados de Inman-Bamber (1994) e Bezuidenhout et al. (2003). Contudo, este padrão difere do observado por Suguitani (2006) e Marin et al. (2011), que analisaram dados coletados no Estado de São Paulo e Mato Grosso do Sul, nos quais as variedades avaliadas não apresentaram pico de perfilhamento tão evidente. Apesar disso, houve boa correlação e concordância no ajuste entre dados simulados e observados, com $\mathrm{R}^{2}=0,892$ e $\mathrm{D}=0,800 . \mathrm{Na}$ variedade CTC 7, o pico de perfilhamento foi superestimado pelo modelo, porém com ajuste relativamente melhor na fase final de maturação, quando o número de perfilhos estabilizouse em torno de 8,0 perfilhos $\mathrm{m}^{-2}$. Os índices estatísticos mostraram correlação entre os dados observados e estimados, com $\mathrm{R}^{2}=0,721$ e $\mathrm{D}=0,827$.

A variedade CTC 20 apresentou a melhor correlação $\left(R^{2}=0,916\right)$ e concordância $(D=0,951)$, mas com pequeno atraso em relação aos dados observados. O pico de perfilhamento ficou em torno dos 120 DAP, com aproximadamente 18 perfilhos $\mathrm{m}^{-2}$ e estabilização em torno de 9 plantas $\mathrm{m}^{-2}$. A variedade RB 86-7515 apresentou pico em torno de 20 plantas $\mathrm{m}^{-2}$, próximo ao dado observado, com rápido decréscimo até a estabilização em 9 perfilhos $\mathrm{m}^{-2}$. Houve leve superestimativa em relação aos dados obtidos, que se estabilizaram em torno de 7,0 perfilhos $\mathrm{m}^{-2}$.
Os índices estatísticos ficaram em $\mathrm{R}^{2}=0,576$ e $\mathrm{D}=0,873$.

As quatro variedades analisadas quanto ao teor de sacarose na matéria fresca (Pol \%) tiveram valores finais próximos aos observados, mas com superestimativa para valores no meio do ciclo. As variedades CTC apresentaram valores de $\mathrm{R}^{2}$ entre 0,506 e 0,807 e de RMSE entre 1,437 e 2,611 (Tabela 3), que se aproximaram dos valores obtidos por O'Leary (2000) $\left(\mathrm{R}^{2}=0,66\right.$ e RMSE $\left.=6,07 \%\right)$ para a variedade sul-africana NCo 376, com uso de versão anterior do DSSAT/Canegro.

O acúmulo de sacarose nas variedades CTC ocorreu no segundo terço do ciclo, em torno de 150 DAP, até aproximadamente 350 DAP, a partir de quando esse acúmulo permaneceu estável. Esse resultado pode ser consequência parcial do tombamento da cultura ocorrido neste período. De modo contrário, a simulação para a variedade RB 83-5486 apresentou acúmulo contínuo de sacarose até a data da colheita. Singels et al. (2010) observaram estabilização no acúmulo de sacarose aproximadamente aos 190 DAP.

As variedades CTC apresentaram número de folhas verdes de 7,0 a 8,0 , com pico a aproximadamente 150 DAP, o que coincide com o observado por Suguitani (2006). Porém, a estimativa dessa variável pelo DSSAT/ Canegro apresentou atraso no surgimento de folhas, quando comparado com os dados observados, o que parece ter sido decorrente de deficiência no ajuste dos parâmetros TTPLNTEM e TTRATNEM. A variedade CTC 4 apresentou boa correlação entre os dados $\left(\mathrm{R}^{2}=0,944\right)$ e concordância razoável $(\mathrm{D}=0,613)$. Já a variedade CTC 20, apresentou correlação regular $\left(\mathrm{R}^{2}=0,740\right)$ e boa concordância $(\mathrm{D}=0,802)$.

A variedade CTC 7 apresentou baixa correlação e concordância $\left(\mathrm{R}^{2}=0,480 ; \mathrm{D}=0,586\right)$, o que também foi verificado por Marin et al. (2011) para as variedades RB 72-454 e SP 83-2847. Conforme mencionado, essa baixa correlação pode ser consequência da concentração na medição dos dados no meio do ciclo da cultura.

Somente a variedade RB 86-7515 apresentou dados como os observados para massa de matéria seca do colmo. Verificou-se superestimativa dessa variável durante todo o ciclo, com estabilização dos dados estimados no final do ciclo. Os índices estatísticos obtidos foram: $\mathrm{R}^{2}=0,534, \mathrm{D}=0,665$ e RMSE $=9,51 \mathrm{Mg} \mathrm{ha}^{-1}$ (Tabela 2), maiores que os obtidos por Singels \& Bezuidenhout (2002) $\left(\mathrm{RMSE}=5,48 \mathrm{Mg} \mathrm{ha}^{-1}\right)$, em 
simulação para a variedade NCo 376, na África do Sul. Entretanto, os resultados são similares aos encontrados por Marin et al. (2011).

O padrão de superestimativa observado nas simulações pode estar relacionado aos dados de solo utilizados. O sistema DSSAT utiliza equações de pedotransferência desenvolvidas para solos americanos que muitas vezes não se adequam aos solos brasileiros. A simulação do estresse hídrico é muito dependente da configuração do solo utilizada. A este respeito, Marin et al. (2011) utilizaram as equações de pedotransferência de Tomasella et al. (2000) para minimizar este problema, o que parece ter sido um procedimento especialmente importante para simulações em solos típicos do Nordeste brasileiro. Outra inferência possível está relacionada ao mecanismo de simulação do estresse hídrico por parte do modelo, que resultou em estresses aquém dos indicados pela cultura, o que possibilitou a explicação apenas parcial da consistente tendência de superestimativa durante boa parte do ciclo da cultura.

\section{Conclusões}

1. O modelo DSSAT/Canegro apresenta melhor capacidade preditiva para as variáveis produtividade, teor de sacarose, altura de colmo e índice de área foliar, quando parametrizado com a técnica Glue, com ajuste visual em conjunto, para evitar perda de processos fisiológicos pela simulação.

2. O modelo é ferramenta promissora para a simulação do crescimento da cultura da cana-de-açúcar, para as variedades brasileiras, quando adequadamente parametrizado.

\section{Referências}

BEZUIDENHOUT, C.N.; O'LEARY, G.J.; SINGELS, A.; BAJIC, V.B. A process-based model to simulate changes in tiller density and light interception of sugarcane crops. Agricultural Systems, v.76, p.589-599, 2003.

COSTA, M.C.G.; MAZZA, J.A.; VITTI, G.C.; JORGE, L.A. de C. Distribuição radicular, estado nutricional e produção de colmos e de açúcar em soqueiras de dois cultivares de cana-de-açúcar em solos distintos. Revista Brasileira de Ciência do Solo, v.31, p.1503-1514, 2007.

GOLDEMBERG, J. Ethanol for a sustainable energy future. Science, v.315, p.808-810, 2007.
INMAN-BAMBER, N.G. A growth model for sugarcane based on a simple carbon balance and the CERES-Maize water balance. South African Journal of Plant Soil, v.8, p.93-99, 1991.

INMAN-BAMBER, N.G. DSSAT/CANEGRO: its history, conceptual basis, present and future uses. In: ROBERTSON, M.J. (Ed.). Research and modelling approaches to assess sugarcane production opportunities and constrains. Brisbane: University of Queensland, 1994. p.31-34.

INMAN-BAMBER, N.G.; KIKER, G. DSSAT/CANEGRO 3.10: DSSAT version 3.1. Honolulu: University of Hawaii, 1997.

JONES, C.A.; KINIRY, J.R. Ceres-maize: a simulation model of maize growth and development. College Station: Texas A \& M University, 1986. 194p.

JONES, C.A.; WEGENER, M.K.; RUSSEL, J.S.; MCLEOD, I.M.; WILLIAMS, J.R. AUSCANE - simulation of Australian sugarcane with EPIC. Brisbane: Commonwealth Scientific and Industrial Research Organization, 1988. 194p.

JONES, J.W.; HE, J.; BOOTE, K.J.; WILKENS, P.; PORTER, C.H.; HU, Z. Estimating DSSAT cropping system cultivar-specific parameters using Bayesian techniques. In: AHUJA, L.R.; MA, L. (Ed.). Methods of introducing system models into agricultural research. Madison: American Society of Agronomy, 2010. p.365-395. (Advances in agricultural systems modeling, 2).

KEATING, B.A.; ROBERTSON, M.J.; MUCHOW, R.C.; HUTH, N.I. Modelling sugarcane production systems I. Development and performance of the sugarcane module. Field Crops Research, v.61, p.253-271, 1999.

KNOX, J.W.; RODRÍGUEZ DÍAZ, J.A.; NIXON, D.J.; MKHWANAZI, M. A preliminary assessment of climate change impacts on sugarcane in Swaziland. Agricultural Systems, v.103, p.63-72, 2010.

LIU, D.L.; KINGSTON, G. QCANE: a simulation model of sugarcane growth and sugar accumulation. In: ROBERTSON, M.J. (Ed.). Research and modelling approaches to assess sugarcane production opportunities and constrains. St. Lucia: University of Queensland, 1995. p.25-29.

MARIN, F.R.; JONES, J.W.; ROYCE, F.; SUGUITANI, C.; DONZELI, J.L.; PALLONE FILHO, W.J.; NASSIF, D.S.P. Parameterization and evaluation of predictions of DSSAT/ CANEGRO for sugarcane Brazilian production systems. Agronomy Journal, v.103, p.100-110, 2011.

MARTINÉ, J.F. Modélisation de la production potentielle de la canne à sucre en zone tropicale, sous conditions thermiques et hidriques contrastées. Applications du modéle. 2003. 130p. Thése (Docteur) - Institut National Agronomique Paris-Gragnon, Paris.

MERTENS, J.; MADSEN, H.; FEYEN, L.; JACQUES, D.; FEYES, $J$. Including prior information in the estimation of effective soil parameters in unsaturated zone modelling. Journal of Hydrology, v.194, p.251-269, 2004.

O'LEARY, G.J. A review of three sugarcane simulation models with respect to their prediction of sucrose yield. Field Crops Research, v.68, p.97-111, 2000. 
PELlEGRINO, G.Q. Utilização de dados espectrais do satélite NOAA14/AVHRR como fonte de dados para modelos matemáticos de estimativa da fitomassa da cana-de-açúcar. 2001. 133p. Tese (Doutorado) - Universidade Estadual de Campinas, Campinas.

RITCHIE, J.T. Soil water balance and plant water stress. In: TSUJ, G.Y.; HOOGENBOOM, G.; THORNTON, P.K. (Ed.). Understanding options for agricultural production. Dordrecht: Kluwer, 1998. p.41-53.

SALTELLI, A. Sensitivity analysis for importance assessment. Risk Analysis, v.22, p.579-590, 2002.

SILVA, L.C. da. Crescimento e acúmulo de nutrientes em sete cultivares de cana-de-açúcar (Saccharum spp.) na região de Coruripe-AL. 2007. 104p. Dissertação (Mestrado) - Universidade Federal de Alagoas, Rio Largo.

SINGELS, A.; BEZUIDENHOUT, C.N. A new method of simulating dry matter partitioning in the Canegro sugarcane model. Field Crops Research, v.78, p.151-164, 2002.

SINGELS, A.; JONES, M.; VAN DER BERG, M. DSSAT v.4.5 DSSAT/CANEGRO: sugarcane plant module: scientific documentation. Mount Edgecombe: International Consortium for
Sugarcane Modeling: South African Sugarcane Research Institute, 2008. 34p.

SINGELS, A.; VAN DER BERG, M.; SMIT, M.A.; JONES, M.R.; VAN ANTWERPEN, R. Modelling water uptake, growth and sucrose accumulation of sugarcane subjected to water stress. Field Crops Research, v.117, p.59-69, 2010.

SUGUITANI, C. Entendendo o crescimento e produção da cana de açúcar: avaliação do modelo Mosicas. 2006. 60p. Tese (Doutorado) - Escola Superior de Agricultura Luiz de Queiroz, Piracicaba.

TOMASELLA, J.; HODNETT, M.G.; ROSSATO, L. Pedotransfer functions for the estimation of soil water retention in Brazilian soils. Soil Science Society of America Journal, v.64, p.327-338, 2000.

VILLEGAS, F.D.; DAZA, O.H.; JONES, J.W.; ROYCE, F.S. CASUPRO: an industry-driven sugarcane model. Tampa: American Society of Agricultural and Biological Engineers, 2005. 22p. (ASAE Annual International Meeting).

WILLMOTT, C.J. Some comments on the evaluation of model performance. Bulletin of the American Meteorological Society, v.63, p.1309-1313, 1982.

Recebido em 26 de julho de 2011 e aprovado em 13 de janeiro de 2012 